Revue internationale P.M.E.

Économie et gestion de la petite et moyenne entreprise

Revuse

internationale

PME

\title{
L'évolution structurelle du commerce de gros français
}

\section{Luuk Klomp et Eugène Rebers}

Volume 8, numéro 2, 1995

URI : https://id.erudit.org/iderudit/1008351ar

DOI : https://doi.org/10.7202/1008351ar

Aller au sommaire du numéro

Éditeur(s)

Presses de l’Université du Québec

ISSN

0776-5436 (imprimé)

1918-9699 (numérique)

Découvrir la revue

Citer cet article

Klomp, L. \& Rebers, E. (1995). L'évolution structurelle du commerce de gros français. Revue internationale P.M.E., 8(2), 99-118.

https://doi.org/10.7202/1008351ar

\section{Résumé de l'article}

Cet article traite de l'évolution structurelle du commerce de gros français. Pour la période 1978-1989, les changements dans la distribution par taille des entreprises et de la productivité du travail ont été analysés pour les trois secteurs principaux. Dans les commerces de gros non alimentaire (secteur 58) et interindustriel (secteur 59), la tendance est à l'accroissement relatif du nombre d'entreprises opérant à faible effectif. Dans le commerce de gros alimentaire (secteur 57), par contre, la taille moyenne a augmenté. Puis, nous avons examiné si l'existence ou non des effets de taille a causé l'évolution observée dans la distribution par taille des entreprises. Nous avons utilisé la productivité du travail comme critère pour les effets de taille. Les économies d'échelle ont augmenté dans tous les trois secteurs principaux du commerce de gros français. Nous nous étions attendu à ce résultat pour le secteur 57, mais non pas pour les secteurs 58 et 59. De plus, les plus fortes augmentations d'économies d'échelle sont observées dans le secteur 57. Cela s'explique par les différences dans les distributions par taille des entreprises entre le secteur 57, d'une part, et les secteurs 58 et 59, d'autre part.
Ce document est protégé par la loi sur le droit d'auteur. L'utilisation des services d’Érudit (y compris la reproduction) est assujettie à sa politique d'utilisation que vous pouvez consulter en ligne.

https://apropos.erudit.org/fr/usagers/politique-dutilisation/ 


\title{
L'évolution structurelle du commerce de gros français ${ }^{1}$
}

\author{
Luuk KLOMP* \\ Université Erasmus Rotterdam \\ Eugène REBERS** \\ Université de Maastricht
}

MOTS CLÉS

\section{Distribution par taille des entreprises - Productivité du travail Économies d'échelle - Canal de distribution Évolution structurelle - Commerce de gros français}

\begin{abstract}
RÉSUMÉ
Cet article traite de l'évolution structurelle du commerce de gros français. Pour la période 1978-1989, les changements dans la distribution par taille des entreprises et de la productivité du travail ont été analysés pour les trois secteurs principaux. Dans les commerces de gros non alimentaire (secteur 58) et interindustriel (secteur 59), la tendance est à l'accroissement relatif du nombre d'entreprises opérant à faible effectif. Dans le commerce de gros alimentaire (secteur 57), par contre, la taille moyenne a augmenté. Puis, nous
\end{abstract}

1. Cet article fait partie d'un programme de recherches du centre CASBEC (Centre for Advanced Small Business Economics) de l'Université Erasmus à Rotterdam.

* Luuk Klomp est assistant-professeur au Département de statistique économique de l'Université Erasmus Rotterdam aux Pays-Bas, titulaire d'un DEA en sciences économiques. Actuellement, il dirige des recherches empiriques dans le domaine de la productivité du travail, des marges bénéficiaires, de l'entrée et sortie et de la croissance des entreprises dans quelques secteurs tertiaires. En 1995, il compte soutenir sa thèse sur ce sujet. Adresse : Erasmus Universiteit Rotterdam, Vakgroep Economische Statistiek, H5-15, Postbus 1738, 3000 DR Rotterdam, Pays-Bas.

** Eugène Rebers est étudiant au doctorat en économie à l'Université de Maastricht, titulaire d'un DEA en sciences commerciales. Il a écrit son mémoire à l'Université Erasmus Rotterdam, sur la productivité du travail dans le commerce de gros français. Actuellement, son centre d'intérêt est le financement des grandes entreprises. Adresse : Rijksuniversiteit Limburg, Vakgroep Financiering, Postbus 616, 6200 MD Maastricht, Pays-Bas.

Les auteurs tiennent à exprimer leur reconnaissance à Martin Carree, François Coulomb, Yvonne Prince, Roy Thurik et aux deux évaluateurs anonymes pour leurs commentaires constructifs à propos de cet article. Ils remercient Paul de Boer, François Coulomb et Carlos Martinez Mongay pour leur aide linguistique en ce qui concerne les traductions. 
avons examiné si l'existence ou non des effets de taille a causé l'évolution observée dans la distribution par taille des entreprises. Nous avons utilisé la productivité du travail comme critère pour les effets de taille. Les économies d'échelle ont augmenté dans tous les trois secteurs principaux du commerce de gros français. Nous nous étions attendu à ce résultat pour le secteur 57 , mais non pas pour les secteurs 58 et 59. De plus, les plus fortes augmentations d'économies d'échelle sont observées dans le secteur 57. Cela s'explique par les différences dans les distributions par taille des entreprises entre le secteur 57, d'une part, et les secteurs 58 et 59, d'autre part.

\begin{abstract}
This paper deals with structural changes in French wholesaling. For the period 1978-1989 developments in both firm size distribution and labour productivity are analyzed for three 2 digit industries. The share of small firms shows an upward trend in the wholesale industries in non-food products (SIC 58) as well as in intermediary products (SIC 59). For food products (SIC 57) we found an increase in average firm size. We then studied whether scale economies is the cause of the observed developments in the firm size distributions. We used labour productivity as a proxy for scale economies. Scale economies increased over time in each 2 digit industry in French wholesaling. This result was expected for SIC 57, but for the SIC's 58 and 59 we expected the contrary. Furthermore, scale economies have increased most in SIC 57. This result can be explained by the differences in the developments in firm size distributions between SIC 57 and SIC's 58 and 59.
\end{abstract}

\title{
RESUMEN
}

En este artículo se analiza la estructura del comercio al por mayor francés. Los cambios observados en la evolución de la distribución por tamaño de las empresas y en la productividad del trabajo entre 1978 y 1989 han sido estudiados para los tres sectores principales. En los comercios no alimentarios al por mayor (sector 58) e interindustriales (sector 59) se observa una tendencia al aumento del número de pequeñas empresas. Sin embargo, en el comercio alimentario al por mayor el tamaño medio ha aumentado. También hemos estudiado si la existencia de efectos escala podría ser la causa de la evolución observada en la distribución por tamaño de las empresas en función de su tamaño. Hemos utilizado la productividad del trabajo como criterio representativo de los efectos escala. Las economías de escala han aumentado en los tres sectores principales del comercio al por mayor en Francia. Este resultado era de esperar en el sector 57, pero no en los sectores 58 y 59. Además, las economías de escala han aumentado más en el sector 57 que en los otros dos. Este hecho puede explicarse por medio de las diferencias en las distribuciones por tamaño de las empresas entre el sector 57, por un lado, y los sectores 58 y 59 , por otro. 


\section{Introduction}

L'importance toujours grandissante des petites entreprises dans l'économie nationale a été décrite dans plusieurs études récentes ${ }^{2}$. On peut l'apprécier à travers les statistiques de nombre d'entreprises, de chiffre d'affaires, d'emploi et de création de nouveaux emplois. Le commerce de gros français est un secteur où les petites entreprises jouent un rôle important. Entre 1978 et 1989 , près de $99 \%$ des entreprises du secteur comptaient au plus 100 employés, soit un peu plus de $70 \%$ de l'emploi total du secteur. Nous considérons que le commerce de gros français est particulièrement intéressant à analyser, non seulement à cause du grand nombre de petites entreprises, mais aussi parce qu'il fait partie du secteur tertiaire. En effet, le secteur tertiaire a peu retenu l'attention dans la tradition de l'organisation industrielle, surtout sur le plan empirique ${ }^{3}$.

Nous nous proposons dans cet article d'analyser l'évolution de la structure du commerce de gros français. Pour la période de 1978 à 1989, nous étudierons, pour les trois secteurs principaux, les changements, d'une part, dans la distribution par taille des entreprises et, d'autre part, dans la productivité du travail. Avec les développements dans la distribution par taille des entreprises, nous abordons la dynamique dans la structure du marché. Pour les entrepreneurs, cette dynamique est importante à reconnaître pour bien comprendre les changements dans l'environnement qui, à leur tour, influencent la stratégie de la firme 4 . Selon Porter (1981), les changements dans l'environnement sont un des quatre éléments fondamentaux pour formuler une stratégie efficace ${ }^{5}$.

Les développements observés dans la distribution par taille des entreprises donnent non seulement une perspective de la dynamique dans la structure du marché, mais aussi du développement de la position des petites et moyennes entreprises par rapport aux grandes entreprises. En même temps, nous examinons à quel point l'évolution de la distribution par taille des entreprises peut être expliquée par des effets de taille. Ces effets seront analysés en reliant la productivité du travail à la taille des entreprises.

Le plan de cet article est le suivant: dans la section 2, nous donnons une brève description des activités et de la fonction du commerce de gros français, notamment la place qu'il tient dans l'économie française. La section 3 constitue

2. Voir, par exemple, Birch (1981 et 1987), Storey et Johnson (1987), Acs et Audretsch (1989), Brock et Evans (1989), Carlsson (1989), Sato (1989) et Schwalbach (1989).

3. Voir, par exemple, Schmalensee (1989) et Davis, Haltiwanger et Schuh (1993).

4. Voir, par exemple, Teece (1984).

5. Porter (1981) précise comment la littérature sur l'organisation industrielle peut contribuer au management stratégique. 
la section empirique. Après une brève description des données, nous examinons dans la sous-section 3.1 les changements sur le plan de la distribution par taille des entreprises. Un sujet qui lui est étroitement lié, la productivité du travail et l'existence ou non des effets de taille, est traité dans la soussection suivante. L'article s'achève par un résumé à la section 4 .

\section{Activités et fonction économique du commerce de gros français}

La description des activités du commerce de gros pose relativement peu de problèmes et on pourrait les décrire comme suit: «la vente des marchandises, qui sont produites autre part, à des revendeurs ou à des utilisateurs professionnels, sans que ces marchandises subissent un changement physique réel » (CBS, 1990). Cependant, le caractère immatériel de ces activités a eu pour conséquence que la fonction économique du commerce de gros n'a pas toujours été appréciée à sa juste valeur (Van Dalen, 1992). Pour bien la comprendre, il est important d'examiner de près le système de production tel que nous le connaissons en Occident.

L'émergence de la production industrielle est allée de pair avec un approfondissement de la division du travail. Un flot de marchandises passe par un canal de distribution dans le but final d'arriver au consommateur. Cependant, avant que les marchandises n'arrivent au bout du canal, elles doivent passer par différents marchés où l'offre et la demande se rencontrent. Une circulation aisée est donc très importante. Le grand nombre et la complexité des marchandises font que les conditions, dans lesquelles un certain membre du canal met en vente ses produits, peuvent différer des conditions auxquelles le second désire les acheter. C'est alors que, quand ces décalages se présentent, le commerce de gros transforme les conditions de vente pour l'un en conditions d'achat pour l'autre. C'est la fonction de transformation du commerce de gros (Van de Woestijne, 1966).

En fonction du type de clientèle et de marchandises, l'INSEE ${ }^{6}$ distingue trois secteurs principaux dans le commerce de gros français:

- 57 commerce de gros alimentaire;

- 58 commerce de gros non alimentaire;

-59 commerce de gros interindustriel.

Du point de vue des marchandises, on distingue l'alimentaire et le nonalimentaire. La clientèle des grossistes se subdivise en deux types, à savoir les

6. Institut national de la statistique et des études économiques. 
revendeurs et les utilisateurs professionnels. Le tableau 1 décrit le poids relatif des deux types de clientèle suivant les trois secteurs principaux.

\section{TABleau 1}

Répartition en pourcentage des ventes en gros par type de clientèle

\begin{tabular}{lcccc}
\hline & $\begin{array}{c}\text { Commerce de } \\
\text { gros alimentaire } \\
\mathbf{( 5 7 )}\end{array}$ & $\begin{array}{c}\text { Commerce de gros } \\
\text { non alimentaire } \\
\mathbf{( 5 8 )}\end{array}$ & $\begin{array}{c}\text { Commerce de gros } \\
\text { interindustriel } \\
\mathbf{( 5 9 )}\end{array}$ & $\begin{array}{c}\text { Ensemble du } \\
\text { commerce de gros }\end{array}$ \\
\hline $\begin{array}{l}\text { Vente à des: } \\
- \text { revendeurs }\end{array}$ & $74,1 \%$ & $65,8 \%$ & $32,7 \%$ & $56,4 \%$ \\
$-\begin{array}{l}\text { utilisateurs } \\
\text { professionnels }\end{array}$ & 25,9 & 34,2 & 67,3 & 43,6 \\
\hline
\end{tabular}

Source : INSEE - enquête annuelle d'entreprise 1987.

Les ventes des secteurs 57 et 58 se font donc principalement à des revendeurs (grossistes, centrales d'achats, détaillants), tandis que pour le secteur 59, les clients les plus importants sont des utilisateurs professionnels (producteurs, collectivités).

Le tableau 2 présente le nombre d'entreprises et de personnes travaillant dans le commerce de gros français. Ils représentent $4,5 \%$ du nombre total d'entreprises et de travailleurs en France.

TABleaU 2

Nombre d'entreprises et nombre d'emplois

\begin{tabular}{rrrrr|rrrr}
\hline \multicolumn{5}{c|}{ Entreprises } & \multicolumn{4}{c}{ Employés } \\
\hline & $\mathbf{5 7}$ & $\mathbf{5 8}$ & $\mathbf{5 9}$ & Total & $\mathbf{5 7}$ & $\mathbf{5 8}$ & $\mathbf{5 9}$ & Total \\
\hline $\mathbf{1 9 7 8}$ & 36986 & 19789 & 24178 & 80953 & 309112 & 257679 & 346603 & 913394 \\
$\mathbf{1 9 7 9}$ & 35003 & 19963 & 24467 & 79433 & 314136 & 275712 & 365184 & 955032 \\
$\mathbf{1 9 8 0}$ & 33257 & 20480 & 24851 & 78588 & 298089 & 268766 & 368574 & 935429 \\
$\mathbf{1 9 8 1}$ & 31354 & 20487 & 24976 & 76817 & 291921 & 261085 & 363395 & 916401 \\
$\mathbf{1 9 8 2}$ & 32379 & 20895 & 24331 & 77605 & 297498 & 26944 & 364153 & 925595 \\
$\mathbf{1 9 8 3}$ & 32646 & 21236 & 26230 & 80112 & 290678 & 261461 & 358455 & 910594 \\
$\mathbf{1 9 8 4}$ & 30200 & 20334 & 26004 & 76538 & 292701 & 257595 & 362675 & 912971 \\
$\mathbf{1 9 8 5}$ & 29291 & 21076 & 27171 & 77538 & 275505 & 258352 & 355417 & 889274 \\
$\mathbf{1 9 8 6}$ & 28022 & 22555 & 26998 & 80406 & 264962 & 259785 & 356891 & 881638 \\
$\mathbf{1 9 8 7}$ & 27846 & 23586 & 28974 & 80406 & 266559 & 260928 & 366780 & 894167 \\
$\mathbf{1 9 8 8}$ & 28219 & 26139 & 30456 & 84406 & 264702 & 273733 & 382858 & 921293 \\
$\mathbf{1 9 8 9}$ & 28771 & 27424 & 34039 & 90234 & 264120 & 282941 & 405698 & 952759 \\
\hline
\end{tabular}

Source : INSEE - enquête annuelle d'entreprise. 
Du tableau 2, on relève que le commerce de gros est globalement un secteur en croissance: en 12 ans, le nombre d'entreprises est passé de 80953 entreprises en 1978 à 90234 entreprises en 1989, soit une augmentation de $11,4 \%$. Quant au nombre de travailleurs, ce pourcentage s'élève à 4,3 . Pour le commerce de gros, on remarquera donc une baisse de la taille moyenne ${ }^{7}$.

Si nous considérons les trois secteurs séparément, il ressort que les commerces de gros non alimentaire (58) et interindustriel (59) prennent une place de plus en plus importante par rapport au commerce de gros alimentaire (57). Cela est dû à l'assortiment des marchandises qui s'est modifié pendant la période 1978-1989, joint à un changement de la fonction du commerce de gros. Nous y reviendrons dans la sous-section 3.1.

\section{Recherche empirique}

Nous disposons d'une banque de données sectorielles pour la période de 1978 jusqu'à 1989. Pour ces douze années, nous avons, pour 10 classes de taille et pour les trois secteurs principaux du commerce de gros français, des données concernant le nombre d'entreprises, le nombre d'emplois occupés et la valeur ajoutée. C'est ainsi que nous disposons de 360 observations, les données provenant d'une vaste enquête annuelle effectuée par l'INSEE auprès des entreprises françaises sous le nom «Enquête annuelle d'entreprise».

\subsection{Distribution par taille des entreprises}

\subsubsection{Les critères}

Nous allons utiliser quatre critères pour étudier les changements dans la distribution par taille des entreprises pendant la période 1978-1989. Premièrement, nous localiserons la distribution par la moyenne $[\mu]$. Puis nous décrirons, comme Carree et Thurik (1991), la forme de la distribution en utilisant l'asymétrie de la distribution [SKEW], le quotient des nombres de petites et grandes entreprises $\left[\mathrm{M}_{\mathrm{PG}}\right]$ et la concentration de la distribution [GINI].

Pour calculer ces critères, nous définissons les variables TE : le nombre total de personnes employées dans un secteur; TF: le nombre total d'entreprises dans un secteur, $f_{i}$ : le nombre d'entreprises dans la classe $i$ divisé par TF, et

7. L'évolution du commerce de gros français est plus ou moins identique à celle de l'ensemble des entreprises françaises. Entre 1979 et 1987, le nombre d'entreprises a augmenté de 9,9\%, tandis que le nombre d'employés, lui, n'a presque pas changé. 
$\mu i$ : le nombre d'emplois dans la classe $i$ divisé par le nombre d'entreprises dans la classe $i^{8}$. Les quatre critères se définissent de la façon suivante:

$$
\mu=\sum_{i=1} f_{i} \mu_{i}=\mathrm{TE} / \mathrm{TF}
$$

La variable $\mu$ représente la localisation de ia distribution. Elle est égale à la taille moyenne des entreprises du secteur considéré. Plus la valeur de $\mu$ est petite, plus la taille moyenne des entreprises est petite, ce qui indique, ceteris paribus, une importance plus grande des petites entreprises

$$
\mathrm{SKEW}=\left(\sum_{i=1}^{10} f_{i} \mu_{i}^{3}-3 \mu \sum_{i=1}^{10} f_{i} \mu_{i}^{2}+2 \mu^{3}\right),\left(\sum_{i=1}^{10} f_{i} \mu_{i}^{2}-\mu^{2}\right) 1,5 .
$$

La variable SKEW représente l'asymétrie de la distribution. Si la distribution est symétrique, SKEW est égale à zéro, tandis que la distribution devient plus asymétrique à mesure que SKEW s'écarte de zéro. Une valeur positive pour SKEW implique une asymétrie à droite, ce qui signifie qu'il y a relativement beaucoup d'entreprises ayant une taille moins élevée que la moyenne et relativement peu d'entreprises ayant une taille plus grande que la moyenne

$$
\mathrm{M}_{\mathrm{PG}}=\sum_{i=1}^{5} f i \quad \stackrel{10}{i=9} \text {. }
$$

La variable $\mathrm{M}_{P G}$ représente le quotient des nombres respectivement des petites et des grandes entreprises. Font partie des catégories de taille 1 à 5 les entreprises ayant moins de 10 salariés et, des catégories 9 à 10 , les entreprises ayant 100 salariés ou plus. Une augmentation de la valeur de $\mathbf{M}_{\mathrm{PG}}$ signifie une augmentation dans la part des petites entreprises par rapport aux grandes entreprises

$$
\mathrm{GINI}=1-(\mathrm{TF} / \mathrm{TE}) \cdot\left(\sum_{i=1}^{10} f_{i}^{2} \mu_{i}+\sum_{j=i+1}^{10} \sum_{i=1}^{9} 2 f_{i} f_{j} \mu_{i}\right) .
$$

8. Il y a 10 classes de taille, classées dans l'ordre ascendant. 
Le coefficient GINI est un critère de concentration basé sur une courbe de concentration. Un exemple d'une telle courbe est présenté dans la figure 1 avec, sur l'axe des $y$, le pourcentage cumulé de l'emploi et, sur l'axe des $x$, le pourcentage cumulé des entreprises.

Le coefficient GINI est alors le quotient de la surface de la partie hachurée dans le triangle en bas à droite, d'une part, et de la surface totale de ce triangle, d'autre part. La valeur se situe donc entre 0 et 1 , une valeur élevée représentant une plus grande concentration dans les emplois occupés.

\subsubsection{Résultats}

Dans le tableau 3, sont décrits, pour les quatre critères, la moyenne, la déviation standard et la variation exprimée en pourcentage pour la période 1978-1989. Les figures 2 jusqu'à 5 suivent les critères d'une année sur l'autre.

Si nous comparons les développements de $\mu$, SKEW et $M_{P G}$ dans le temps, il semble qu'une baisse de $\mu$ aille de pair avec une hausse de SKEW et de $M_{P G}$, et vice versa. La relation entre ces trois critères résulte du fait qu'ils décrivent tous les trois l'importance relative des petites entreprises par rapport aux grandes entreprises. Cependant, la valeur qu'on peut accorder aux changements des critères est plus grande au fur et à mesure que la relation entre ces changements est plus forte. En ce qui concerne les trois critères $\mu$, SKEW et $\mathrm{M}_{\mathrm{PG}}$, les trois secteurs se sont rapprochés pendant la période 1978-1989. On peut en tirer la conclusion que les différences dans les distributions par taille des entreprises ont diminué. Le coefficient GINI connaît une faible augmentation dans le temps. Le degré de concentration dans le commerce de gros français a donc augmenté légèrement entre 1978 et 1989, mais il continue d'être assez faible?

Si on compare les trois secteurs mutuellement, on remarque que la distribution par taille des entreprises pour le commerce de gros alimentaire (57) n'est pas conforme aux distributions par taille des entreprises des commerces de gros non alimentaire (58) et interindustriel (59). Dans le secteur 57, la taille moyenne $[\mu]$ est presque stationnaire, tandis que l'asymétrie de la distribution [SKEW] montre aussi un développement peu systématique. Cependant, du fait

9. Nous avons aussi calculé les coefficients GINI à base de quatre classes de taille, de sorte qu'on puisse les comparer à Carree et Thurik (1991). Cette adaptation a causé une baisse d'environ 0,11 , de sorte que la valeur du coefficient GINI montait à environ 0,61 en moyenne pour les trois secteurs. Pour la période 1978-1989, les degrés de concentration dans les commerces de gros français et néerlandais diffèrent peu et ils indiquent, comparés aux autres entreprises néerlandaises, un degré de concentration assez modeste. 


\section{TABlEAU 3}

Moyenne, déviation standard et variation (en \%) pour la période 1978-1989

\begin{tabular}{|c|c|c|c|c|c|c|}
\hline & & $\mu$ & & & SKEW & \\
\hline & moyenne & $\begin{array}{l}\text { déviation } \\
\text { standard }\end{array}$ & $\begin{array}{c}\text { variation } \\
\text { (en \%) }\end{array}$ & moyenne & $\begin{array}{l}\text { déviation } \\
\text { standard }\end{array}$ & $\begin{array}{c}\text { variation } \\
\text { (en \%) }\end{array}$ \\
\hline 57 & 9,37 & 0,32 & $+8,44$ & 12,04 & 0,28 & $-5,88$ \\
\hline 58 & 12,34 & 1,05 & $-20,88$ & 11,61 & 0,41 & $-8,86$ \\
\hline 59 & 13,93 & 1,01 & $-16,59$ & 10,01 & 0,42 & $+9,53$ \\
\hline & moyenne & $\begin{array}{c}\mathbf{M}_{\mathbf{P G}} \\
\text { déviation } \\
\text { standard }\end{array}$ & $\begin{array}{c}\text { variation } \\
(\text { en } \%) \\
\end{array}$ & moyenne & $\begin{array}{c}\text { GINI } \\
\text { déviation } \\
\text { standard }\end{array}$ & $\begin{array}{c}\text { variation } \\
(\text { en \%) }\end{array}$ \\
\hline 57 & 88,58 & 7,85 & $-24,09$ & 0,729 & 0,009 & $+3,41$ \\
\hline 58 & 66,28 & 7,51 & $+40,87$ & 0,717 & 0,014 & $+4,75$ \\
\hline 59 & 50,31 & 6,53 & $+36,46$ & 0,715 & 0,007 & $-0,55$ \\
\hline
\end{tabular}

FIGURE 1

Courbe de concentration

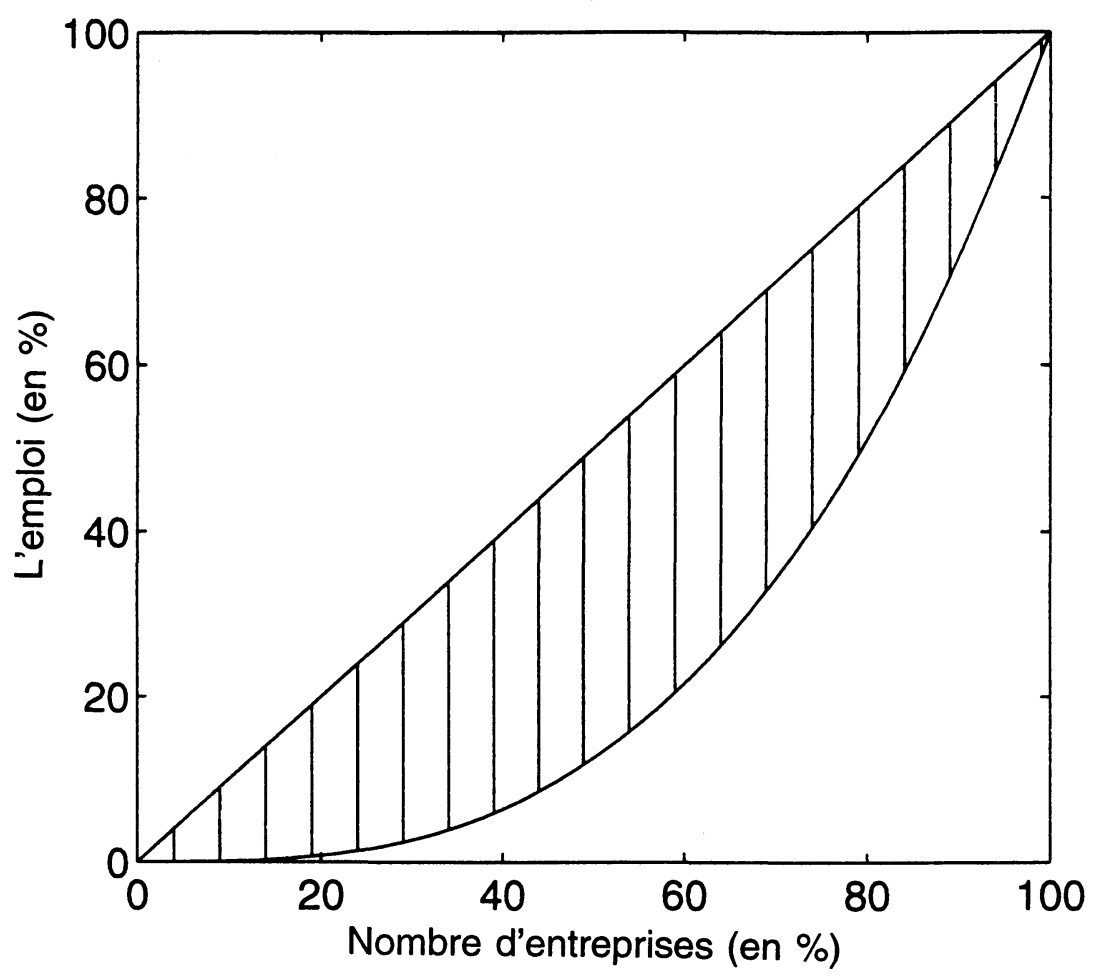

Revue internationale P.M.E., vol. 8, nº 2, 1995 
Figure 2

Évolution de $\mu$

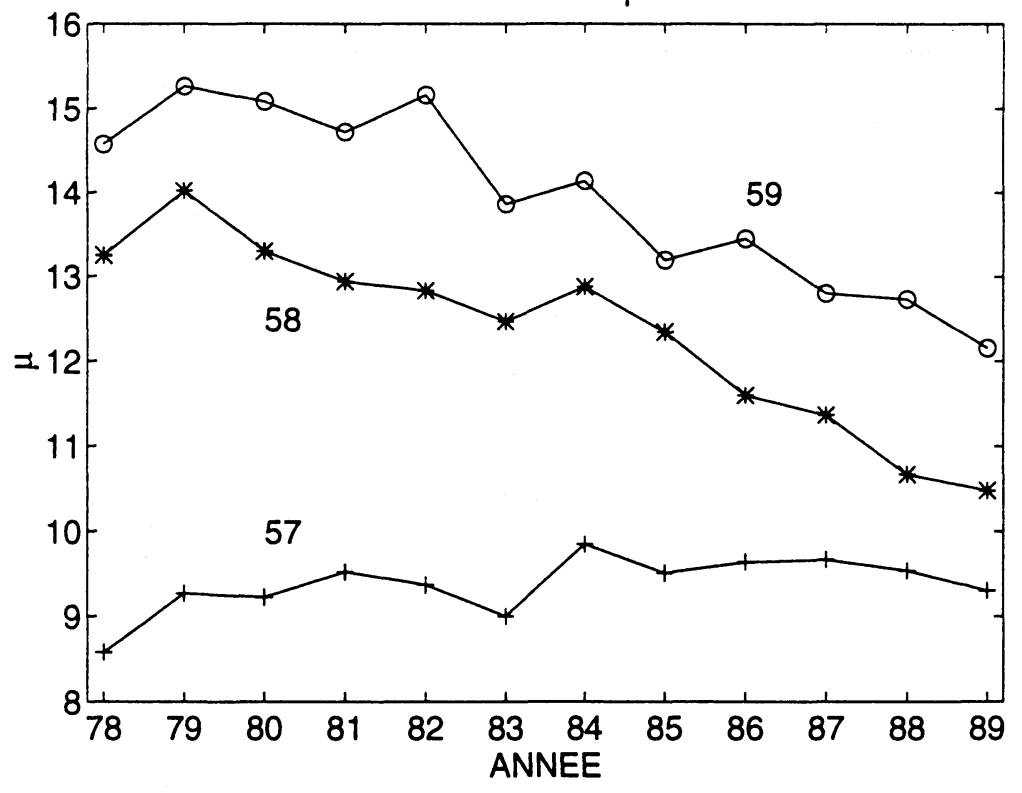

FIGURE 3

Évolution de SKEW

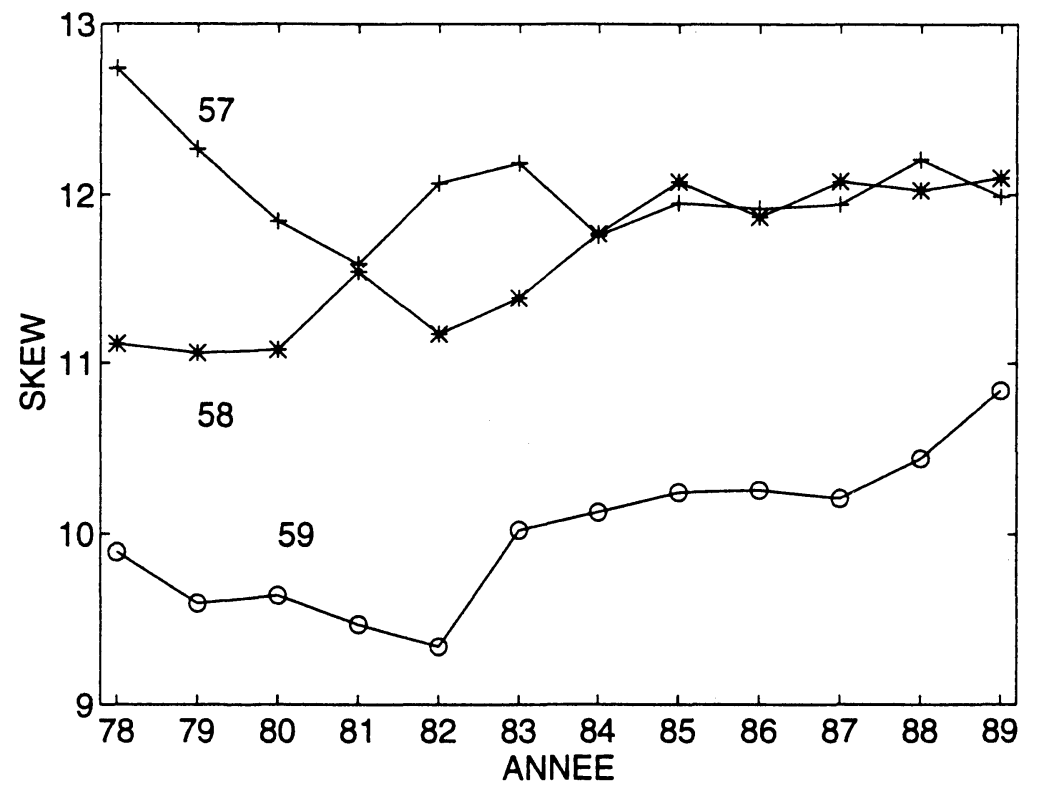


Figure 4

Évolution de MPG

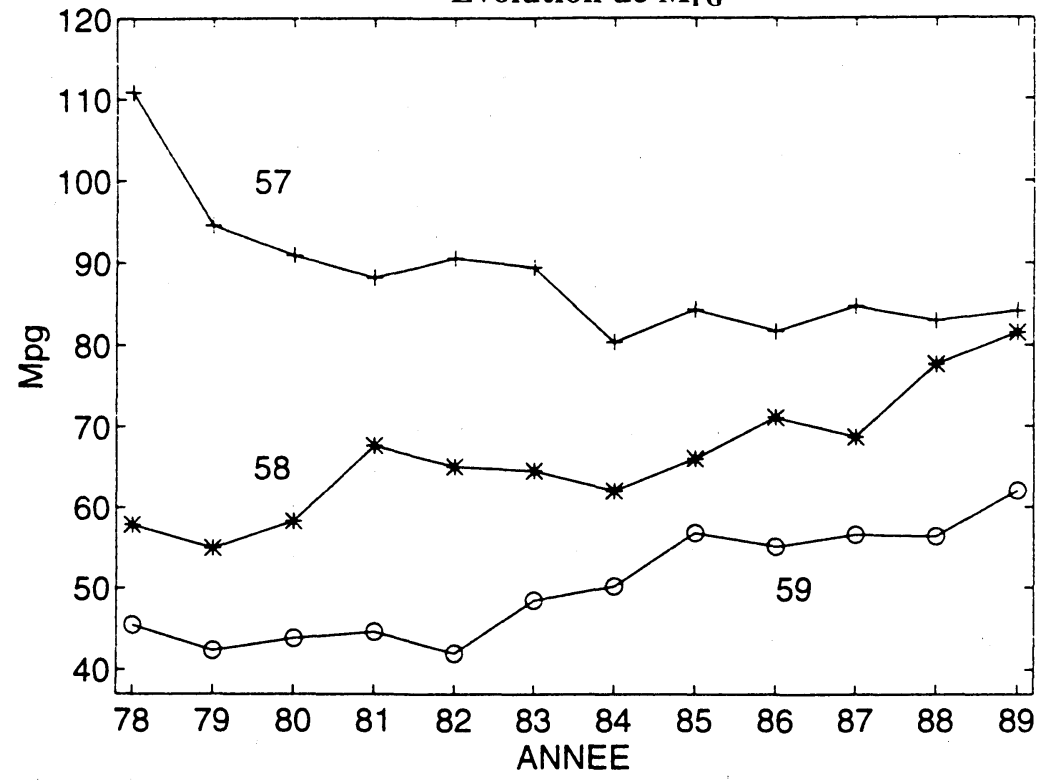

Figure 5

Évolution de GINI

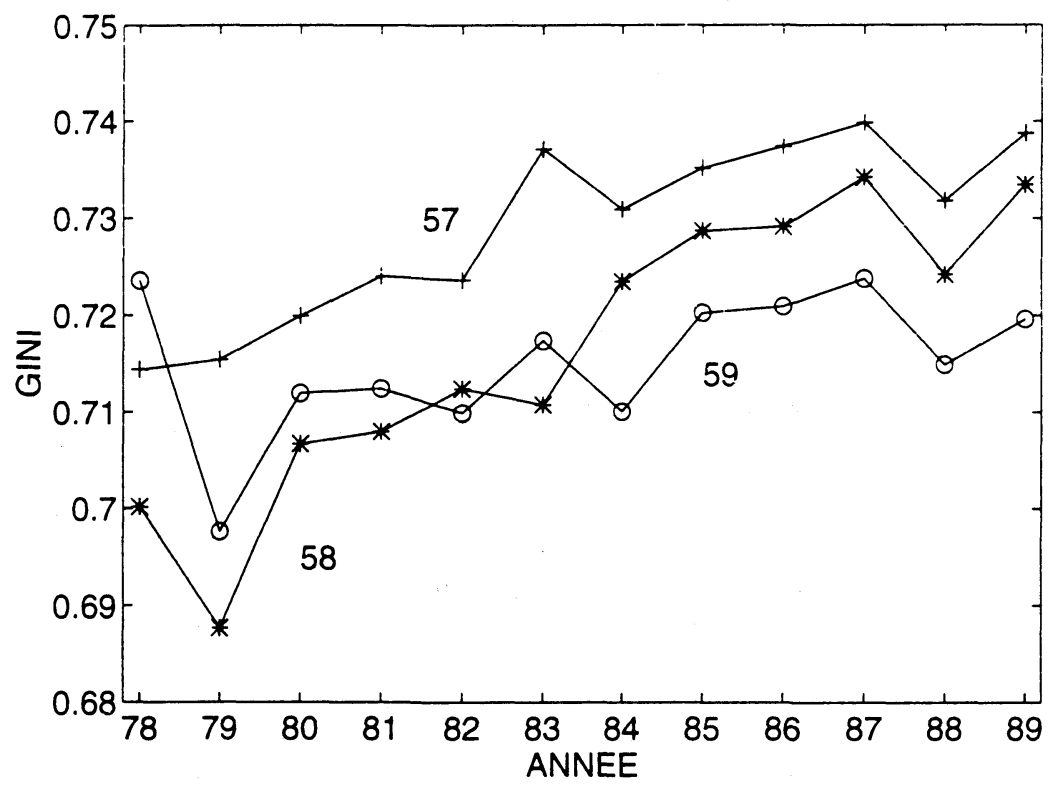


du développement de $\mathrm{M}_{\mathrm{PG}}$, il semble que l'importance des petites entreprises a diminué par rapport aux grandes entreprises.

Les changements de la distribution par taille des entreprises des commerces de gros non alimentaire (58) et interindustriel (59) sont si étroitement liés qu'on peut les prendre en considération indifféremment. Il ressort à la fois d'une diminution de la taille moyenne $[\mu]$ et d'une augmentation des valeurs de SKEW et de $M_{P G}$ que pour ces deux secteurs, l'importance des petites entreprises a augmenté par rapport aux grandes entreprises dans la période 1978-1989. Nous estimons, cependant, les différences du coefficient GINI pour les trois secteurs trop faibles pour en tirer d'autres conclusions.

En résumé, on constate que dans les commerces de gros non alimentaire (58) et interindustriel (59), la tendance est à l'accroissement relatif du nombre d'entreprises opérant à faible effectif ${ }^{10}$. Nous supposons que la forte augmentation du nombre des (petites) entreprises est due à l'intensification de la demande des produits spécialisés que les entrepreneurs ont exploitée par l'innovation de nouveaux produits ${ }^{11,12}$.

Le raisonnement ci-dessus ne convient pas au commerce de gros alimentaire (57). La raison en est que le secteur alimentaire se caractérise par un éventail d'articles plutôt homogène qui subit relativement peu de fluctuations. Dans ce secteur, un accroissement de l'effectif moyen s'est développé. En raison d'une forte intégration verticale (centrales d'achats, chaînes de magasins), le commerce de gros a été plus ou moins forcé d'aller opérer lui aussi à une plus grande échelle. Les grandes entreprises ont passablement su

10. Dans la période 1978-1989, le nombre d'entreprises dans les secteurs 58 et 59 a augmenté respectivement de 39 et $40 \%$. Pour les petites entreprises, ces pourcentages s'élèvent à 52 et 48 et, pour les grandes entreprises, à 8 et 8 . L'accroissement du nombre d'entreprises est donc beaucoup plus favorable aux petites qu'aux grandes entreprises.

11. Les données au niveau 4 de la nomenclature nous ont montré que les secteurs «parfumerie et produits de beauté » ainsi que "matériel mobilier de bureau » ont connu une forte augmentation du nombre d'entreprises, tandis que leur taille moyenne a baissé. C'est justement dans ces secteurs qu'il y a un commerce considérable de nouveaux produits.

12. L'intensification de la demande des produits spécialisés et l'innovation de nouveaux produits sont deux des six raisons que nous donnent Brock et Evans (1989) comme explication possible pour la diminution de la taille moyenne des entreprises commencée à la fin des années 1970 aux États-Unis. 
se maintenir dans cette évolution, mais l'ensemble du commerce de gros alimentaire a dû céder du terrain ${ }^{13}$.

La demande des produits spécialisés est avantageux pour les petites entreprises. Grâce à leur flexibilité, elles peuvent répondre rapidement aux variations de la demande par l'introduction de nouveaux produits ${ }^{14}$. Nous estimons que, pour la période 1978-1989, l'importance augmentée de la flexibilité de la production et des services complémentaires dans les commerces de gros non alimentaire (58) et interindustriel (59) montre une diminution des économies d'échelle. Par contre, dans le commerce de gros alimentaire (57), la taille moyenne a augmenté et nous nous y attendions à une augmentation des économies d'échelle.

Dans la sous-section 3.2, nous examinons si l'évolution des effets de taille peut expliquer les changements observés de la distribution par taille des entreprises dans les trois secteurs principaux du commerce de gros français. Nous analysons les effets de taille en reliant, pour la période 1978-1989, l'évolution de la productivité aux classes de taille. Faute de données en ce qui concerne le capital, nous nous limitons à la productivité du travail.

Pour deux raisons, le manque de données relativement au facteur capital n'est pas obligatoirement une grande omission. Premièrement, les entreprises créent l'ensemble de leurs biens d'équipement régulièrement au cours des années, de sorte que le capital reste assez constant d'une année à l'autre. Deuxièmement, les secteurs tertiaires, dont le commerce de gros fait partie, ont un fort coefficient de travail, ce qui se traduit, par exemple, par un niveau $d^{\prime}$ investissements relativement faible ${ }^{15}$. Il semble donc légitime de supposer que les changements dans la productivité sont surtout le résultat des changements dans la productivité du travail.

13. Dans la période 1978-1989, le nombre d'entreprises du commerce de gros alimentaire a diminué de $21 \%$. Pour les petites entreprises, ce pourcentage s'élève à 22 , tandis que les grandes entreprises ont connu une augmentation de $2,5 \%$. La position des grandes entreprises s'est donc beaucoup améliorée par rapport aux petites entreprises.

14. Voir Mills et Schumann (1985) et Julien (1993) pour une discussion sur la flexibilité des petites entreprises.

15. Pendant la période 1978-1989, les investissements dans le commerce de gros français ne représentent que $1,3 \%$ de la valeur ajoutée. 


\subsection{Productivité du travail}

\subsubsection{Le modèle}

Pour l'analyse de la productivité du travail, on construit une équation avec le volume de main-d'œuvre comme variable dépendante et la production comme variable indépendante. Pour la production, nous utiliserons la valeur ajoutée ( $Q$, en millions de francs de 1989) et pour le travail le nombre des personnes occupées $(\mathrm{L})^{16}$. Pour les deux, nous avons calculé la moyenne pour le nombre d'entreprises par classe de taille dans un secteur. Nous proposons l'équation suivante ${ }^{17}$ :

$$
\ln \mathrm{L}=\beta_{\mathrm{o}}+\beta_{1} 1 n \mathrm{Q}
$$

avec: $\ln \mathrm{L}=$ le logarithme népérien du nombre des personnes occupées

$\ln \mathrm{Q}=$ le logarithme népérien de la valeur ajoutée

L'élasticité du travail est alors:

$$
\frac{d \mathrm{~L}}{d \mathrm{Q}} \frac{\mathrm{Q}}{\mathrm{L}}=\frac{d \ln \mathrm{L}}{d \ln \mathrm{Q}}=\beta_{1}
$$

Dans ce modèle, les effets de taille sont donc entièrement déterminés par $\beta_{1}$. Ces effets deviennent plus forts à mesure que $\beta_{1}$ s'écarte de $1 ; \beta_{1}>1$ signifie des déséconomies d'échelle, $B_{1}<1$ signifie des économies d'échelle. Le coefficient $\beta_{0}$ donne une indication de la productivité du travail autonome, c'est-à-dire la productivité du travail indépendante du niveau de production. Puisque nous sommes spécialement intéressés aux développements dans le temps, nous introduisons une variable de temps [T] dans l'équation:

$$
1 n \mathrm{~L}=\beta_{\mathrm{o}}+\tau_{\mathrm{o}} \mathrm{T}+\beta_{1} \ln \mathrm{Q}+\tau_{1} \mathrm{~T} 1 n \mathrm{Q}
$$

avec: $\mathrm{T}=1, \ldots, 12$ pour les années $1978, \ldots, 1989$.

Les coefficients $\tau_{0}$ et $\tau_{1}$ indiquent pour la période 1978-1989 les changements respectivement de la productivité du travail autonome et des effets de taille.

16. La productivité du travail se définit comme la valeur ajoutée $(\mathrm{Q})$ divisée par le nombre des personnes occupées $(\mathrm{L})$.

17. Voir Van Dalen, Koerts et Thurik (1989) pour une discussion de quelques équations de travail. 


\subsubsection{Résultats}

Dans le tableau 4 sont groupés les résultats de l'équation (7). Nous avons appliqué une analyse de régression en utilisant la méthode des moindres carrés et en corrigeant pour l'hétéroscédasticité ${ }^{18}$. L'équation a été estimée pour la période 1978-1989, pour 10 classes de taille par an, soit 120 observations par secteur.

\section{TABleau 4}

Résultats de régression de l'équation :

$\ln \mathrm{L}=\beta_{0}+\tau_{0} \mathrm{~T}+\beta_{1} 1 n \mathrm{Q}+\tau_{1} \mathrm{~T} 1 n \mathrm{Q}$

\begin{tabular}{cccc}
\hline & 57 & 58 & 59 \\
\hline$\beta_{0}$ & 1,1245 & 1,3719 & 1,2992 \\
& $(0,0452)$ & $(0,0286)$ & $(0,0203)$ \\
$\tau_{0}$ & $-0,0023$ & $-0,0188$ & $-0,0135$ \\
& $(0,0061)$ & $(0,0039)$ & $(0,0028)$ \\
$\beta_{1}$ & 1,0674 & 0,9602 & 0,9579 \\
& $(0,0199)$ & $(0,0110)$ & $(0,0082)$ \\
$\tau_{1}$ & $-0,0169$ & $-0,0062$ & $-0,0063$ \\
& $(0,0025)$ & $(0,0015)$ & $(0,0011)$ \\
$\mathbf{R}^{2}$ & 0,9903 & 0,9965 & 0,9981 \\
$\mathbf{n}$ & 120 & 120 & 120 \\
\hline
\end{tabular}

Note : Entre les parenthèses sont indiqués les écarts types.

En comparant les résultats entre les trois secteurs principaux, un phénomène analogue à celui observé dans la distribution par taille des entreprises se présente. Les résultats du secteur 57 diffèrent des résultats des secteurs 58 et 59 , tandis que ces deux derniers ne présentent presque pas de différence entre eux.

Dans le commerce de gros alimentaire (57), il y a initialement des déséconomies d'échelle $\left(B_{1}>1\right)$. Cependant, au cours de la période 1978-1989, ces déséconomies diminuent $\left(\tau_{1}<0\right)$ et se transforment même en économies

18. Nous avons estimé l'équation (7) en deux étapes. Dans une première étape, nous avons calculé les résidus de l'équation (7) selon la méthode des moindres carrés. À la deuxième étape, nous avons pondéré les observations de chaque classe de taille avec la réciproque de l'écart quadratique moyen des résidus de cette classe de taille pendant la période 1978-1989. Puis nous avons estimé l'équation (7) une deuxième fois. Cette procédure ressemble à celle de Prince et Thurik (1993), où les estimations ont été corrigées pour tenir compte de l'hétéroscédasticité et de l'autocorrélation. Nous n'avons pas corrigé pour l'autocorrélation, puisque nous sommes justement intéressés aux développements dans le temps. 
d'échelle ${ }^{19}$. Ce développement des effets de taille est cohérent avec les résultats de la sous-section 3.1 , où nous avions constaté que la position des grandes entreprises s'était améliorée par rapport à celle des petites entreprises. Dans le secteur 57, on n'enregistre pas d'augmentation autonome de la productivité du travail ${ }^{20}$.

Les commerces de gros non alimentaire (58) et interindustriel (59) se caractérisent en 1978 par des économies (légères) d'échelle $\left(B_{1}<1\right)$, économies qui augmentent au cours de la période 1978-1989 $\left(\tau_{1}<0\right)^{21,22}$. Les deux secteurs ont connu également une augmentation autonome de la productivité du travail ${ }^{23}$. Pour les secteurs 58 et 59 , nous conclurons que l'importance grandissante des petites entreprises, observée à la sous-section 3.1, ne s'est pas accompagnée d'une progression supplémentaire de la productivité du travail dans ces entreprises. Au contraire, l'accroissement de la productivité du travail dans les secteurs 58 et 59 est plus favorable aux grandes qu'aux petites entreprises ${ }^{24}$.

Une explication possible pour l'augmentation des économies d'échelle dans les secteurs 58 et 59 est que les grandes entreprises se rendent compte de l'intensification de la demande de produits spécialisés et de l'incertitude de leur débit des produits standardisés. On peut essayer de maintenir le débit par une réduction des prix. Les grandes entreprises peuvent faire attention aux augmentations de la productivité comme possibilité de maintenir leurs marges et de réduire leurs prix en même temps.

19. En 1989 , T prend la valeur de 12 , d'une manière que $\left(\beta_{1}+12 \tau_{1}\right)$ est plus petit que 1 , notamment 0,86 . Les données suivantes montrent clairement ce changement en faveur des grandes entreprises : en 1978, la productivité du travail des petites entreprises est environ $30 \%$ plus grande que celle des grandes entreprises, tandis qu'en 1989 la productivité des grandes entreprises est à peu près $50 \%$ plus grande que celle des petites entreprises.

20. Bien que pendant la période 1978-1989 l'augmentation de la productivité du travail dans le secteur 57 s'élève à presque $45 \%$, elle a diminué de $7,5 \%$ pour les petites entreprises. La productivité du travail des grandes entreprises a presque doublé.

21. Dans les secteurs 58 et 59 , la productivité du travail des grandes entreprises en 1978 est respectivement $20 \%$ et $26 \%$ plus grande que celle des petites entreprises. En 1989, ces pourcentages s'élèvent à 36 et 72 .

22. En 1989, les économies d'échelle pour les secteurs 57,58 et 59 sont presque aussi grandes.

23. L'augmentation de la productivité du travail dans les secteurs 58 et 59 s'élève à respectivement $40 \%$ et $38 \%$.

24. Pendant la période 1978-1989, les petites comme les grandes entreprises des secteurs 58 et 59 ont connu une augmentation de la productivité du travail. Pour les petites et grandes entreprises, ces pourcentages s'élèvent, pour le secteur 58 , à respectivement 26 et 44 ; pour le secteur 59, à respectivement 15 et 57 . 
Si nous comparons l'évolution dans le temps de la productivité des petites et des grandes entreprises pour les trois secteurs, on constate que l'évolution de la productivité des grandes entreprises est plus favorable dans le commerce de gros alimentaire (57) que dans les commerces de gros non alimentaire (58) et interindustriel (59), tandis que c'est l'inverse pour les petites entreprises ${ }^{25}$. L'évolution de la distribution par taille des entreprises, observée dans la sous-section 3.1, nous a montré un accroissement de la taille moyenne dans le secteur 57 et une augmentation de l'importance des petites entreprises dans les secteurs 58 et 59 . L'hypothèse que l'évolution de la distribution par taille des entreprises a été causée par des effets de taille plus forts dans le secteur 57 que dans les secteurs 58 et 59 est donc affirmée par l'évolution de la productivité des petites et grandes entreprises.

\section{Conclusion}

Pour la période de 1978 à 1989, les changements dans la structure du commerce de gros français ont été examinés. Les changements structurels ont été abordés par les développements dans la distribution par taille des entreprises et dans la productivité du travail. Nous avons insisté sur les différences entre les PME et les grandes entreprises. Par les développements dans la distribution par taille des entreprises, nous avons analysé la dynamique dans la structure du marché du commerce de gros français. Cette dynamique est un élément important de l'environnement de la firme et elle peut être utilisée en déterminant la stratégie de la firme. Par les développements dans la productivité du travail, nous avons examiné dans quelle mesure des effets de taille peuvent expliquer les développements observés dans la distribution par taille des entreprises.

Les changements dans la distribution par taille des entreprises et dans la productivité du travail ont été analysés pour les trois secteurs principaux du commerce de gros français, à savoir le commerce de gros alimentaire (57), non alimentaire (58) et interindustriel (59). Les données calculées dans le secteur 57 s'écartent considérablement de celles des secteurs 58 et 59 , les deux derniers étant proches.

Le secteur alimentaire se caractérise par un éventail d'articles plutôt homogène qui subit relativement peu de fluctuations. Dans ce secteur, un processus d'augmentation de taille s'est développé. Le commerce de gros alimentaire (57) suit ce processus surtout par une baisse du nombre des petites entreprises. De plus, on note que le développement de la productivité du travail est beaucoup moins favorable aux petites qu'aux grandes entreprises. En 1978,

25. Pour le secteur $57, \tau_{1}$ est plus négatif que pour les secteurs 58 et 59 . 
la productivité du travail des petites entreprises est plus grande que celle des grandes entreprises, tandis qu'en 1989 on observe la situation inverse. On en conclut que, pour le commerce de gros alimentaire, la position des petites entreprises s'est détériorée par rapport à celle des grandes entreprises.

Du développement de la distribution par taille des entreprises, on déduit que les commerces de gros non alimentaire (58) et interindustriel (59) tendent à produire avec un effectif moyen plus faible. Il est possible que, à la suite d'un éventail d'articles transformé et agrandi, les grossistes se soient spécialisés dans certaines niches. Une position plus forte des petites entreprises par rapport aux grandes entreprises ne s'exprime pas, dans les secteurs 58 et 59, par le développement de la productivité du travail. Au contraire, la productivité du travail augmente avec la taille des entreprises et, au cours de la période 1978-1989, cet effet s'est encore amplifié.

Tous les trois secteurs principaux du commerce de gros français montrent une augmentation des économies d'échelle dans le temps. Donc, dans ces secteurs, la productivité du travail connaît une évolution qui est plus favorable aux grandes qu'aux petites entreprises. Cependant, si nous comparons l'évolution de la productivité du travail des petites et des grandes entreprises, nous observons que la productivité du travail des grandes entreprises s'est développée le plus favorablement dans le secteur 57 et dans les secteurs 58 et 59 pour les petites entreprises. Dans le temps, les économies d'échelle des grandes entreprises se sont donc développées plus intensément dans le secteur 57 que dans les secteurs 58 et 59 . Ces résultats pourront être à l'origine de l'évolution de la distribution par taille des entreprises dans les trois secteurs principaux. La taille moyenne des entreprises a diminué dans le secteur 57 et augmenté dans les secteurs 58 et 59 .

Cet article nous renseigne sur la dynamique des structures du marché du commerce de gros français. Nous avons été contraints d'analyser des groupes d'entreprises. Nous sommes d'avis que des recherches futures devraient être concentrées au niveau de l'entreprise. Des données sur l'entrée, la sortie et sur la croissance des entreprises dans le temps peuvent montrer comment des entreprises de différentes tailles contribuent à la création et à la disparition de l'emploi. Ces données peuvent également signaler les points forts et les points faibles des entreprises qui, à leur tour, peuvent être utilisées dans l'établissement de la stratégie de la firme. À mesure que des données au niveau de l'entreprise sont disponibles, on peut construire un «système de support à la décision » pour aider l'entrepreneur. Par ailleurs, des données sur la structure du marché pourraient y être intégrées. 


\section{Bibliographie}

Acs, Z.J. et D.B. AudRETSCH (1989), «Editors' introduction », Small Business Economics, vol. 1, p. 1-5.

BIRCH, D.L. (1981), «Who creates jobs?», The Public Interest, vol. 65, p. 3-14.

BIRCH, D.L. (1987), Job Creation in America, New York, The Free Press.

Brock, W.A. et D.S. Evans (1989), «Small business economics», Small Business Economics, vol. 1, p. 7-20.

CARLSSON, D.L. (1989), « The evolution of manufacturing technology and its impact on industrial structure : an international study », Small Business Economics, vol. 1, p. 21-37.

CARree, M. et R. ThuriK (1991), "Recent developments in the dutch firm-size distribution», Small Business Economics, vol. 3, p. 261-268.

CBS (1990), Samenvattend Overzicht Groothandel, Voorburg.

Davis, S.J., J. HaltiwANGer et S. SCHUH (1993), Small Business and Job Creation: Dissecting the Myths and Reassessing the Facts, NBER Document de travail 4492.

INSEE (1980-1991), Résultats de l'enquête annuelle d'entreprise, les entreprises du commerce et de l'industrie (plusieurs années), Paris.

JULIEN, P.A. (1993), «Small business as a research subject: some reflections on knowledge of small business and its effects on economic theory », Small Business Economics, vol. 5, p. 157-166.

MiLlS, D.E. et L. SCHUMANN (1985), «Industry structure with fluctuating demand», American Economic Review, vol. 75, p. 758-767.

PORTER, M.E. (1981), «The contributions of industrial organization to strategic management », Academy of Management Review, vol. 6, p. 609-620.

Prince, Y. et R. ThURIK (1993), «Firm-size distribution and price-cost margins in Dutch manufacturing», Small Business Economics, vol. 5, p. 173-186.

SATo, Y. (1989), « Small business in Japan : a historical perspective », Small Business Economics, vol. 1, p. 121-128.

SCHMALENSEE, R. (1989), «Interindustry studies of structure and performance», dans R. Schmalensee et R.D. Willig (éd.), Handbook of Industrial Organization, Amsterdam, Elsevier Science Publishers.

SCHWALBACH, J. (1989), «Small business in German manufacturing », Small Business Economics, vol. 1, p. 129-136.

Storey, D.J. et S. JohnSON (1987), Job Generation and Labour Market Change, London, MacMillan. 
TEECE, D.J. (1984), «Economic analysis and strategic management », California Management Review, vol. 26, $\mathrm{n}^{\circ} 3$, p. 87-110.

THURIK, A.R. (1993), "Recent developments in the firm-size distribution and economics of scale in Dutch manufacturing ", dans Z.J. Acs et D.B. Audretsch (éd.), Small Firms and Entrepreneurship: An East-West Perspective, Cambridge University Press.

van Dalen, J., J. Koerts et A.R. Thurik (1989), An Analysis of Labour Productivity in Wholesaling, Research Institute for Small and Medium Sized Business in the Netherlands, Zoetermeer.

VAN DALEN, J. (1992), «Quantitative Studies in Wholesaling », Thèse de doctorat, Rotterdam.

VAN DE WOESTIJNE, W.J. (1966), «De taak van de tussenhandel - 40 jaar later », dans De Funktie Van de Groothandel, Internatio Stichting, Rotterdam. 